\title{
An Unusual Case of Aneurysmal Bone Cyst Involving Both Middle and Posterior Fossa Skull Base
}

\author{
Shonali Valsangkar ${ }^{1}$ Hrushikesh Kharosekar ${ }^{1}$ Vernon Velho ${ }^{1}$ \\ ${ }^{1}$ Department of Neurosurgery, Sir J. J. Group of Hospitals and Grant \\ Medical College, Mumbai, Maharashtra, India \\ Address for correspondence Shonali Valsangkar, DNB, Department \\ of Neurosurgery, 4th Floor, Sir J. J. Group of Hospitals and Grant \\ Medical, Byculla E, Mumbai 400008, Maharashtra, India \\ Indian J Neurosurg 2016;5:114-116. \\ (e-mail: hkharosekar@gmail.com).
}
Abstract
Keywords
- aneurysmal bone cyst
- middle cranial fossa
- posterior cranial fossa
- clivus

Aneurysmal bone cyst (ABC) is a benign osteolytic bony lesion that can be locally destructive. The skull base is an uncommon location for this tumor with a reported incidence of 3 to $6 \%$. In the literature, we found only 11 cases of skull base $A B C$ reported till 2010, with only 2 involving the posterior cranial fossa skull base. This is the first case involving both the middle and posterior cranial fossa skull base reported till date.

\section{Introduction}

Aneurysmal bone cyst $(\mathrm{ABC})$ is a benign osteolytic bony lesion that can be locally destructive. It is usually observed in the first and second decades of life. This expansile bone lesion commonly involves the metaphysis of long tubular bones, posterior elements of the vertebrae, and flat bones. The skull base is an uncommon location for this tumor with a reported incidence of 3 to $6 \%$. In the literature, we found only 11 cases of skull base ABC reported till 2010, with only 2 involving the posterior cranial fossa skull base. ${ }^{1}$

\section{Case Presentation}

A 22-year-old female patient presented to us with difficulty in swallowing for the past 7 days. She also had headache and imbalance while walking for the past 1 month. On neurological examination, right lateral rectus palsy, right facial deviation, and gag reflex were depressed on the same side. On computed tomography (CT) scan, a large $7 \times 4 \times 9 \mathrm{~cm}$ lobulated extra-axial lytic expansile lesion was seen involving the middle and posterior cranial fossa in midline and on the right side, with multiple fluid-fluid levels within, suggestive of $A B C$ ( - Fig. 1 ). On magnetic resonance imaging (MRI) of the brain with contrast, similar findings were noted with fluid hyperintense on T1-weighted and isointense on T2-weighted images. Septa and cyst wall showed enhancement on contrast. The lesion was causing destruction of basisphenoid, clivus, occipital bone, right temporal bone, jugular foramen, and atlas ( - Fig. 2A,B). The patient was operated in two stages: first endonasal and then retrosigmoid approach for near-total excision of lesion (-Fig. 3). Histopathology showed multiple blood-filled cystic cavities and many multinucleated giant cells. Histopathological features and immunohistochemical analysis were in favor of ABC. Postoperative course was uneventful; patient was advised radiotherapy for residual lesion.

\section{Discussion}

$A B C$ is a benign, nonneoplastic lesion, occurring most commonly before the age of 20 years. ${ }^{1}$ ABC usually involves the metaphysis of the long bones (60\%) and the vertebrae (20\%). Only a few sporadic cases have been reported to have the lesion at the skull base ${ }^{1}$

Clinical presentation of $\mathrm{ABCs}$ depends on the site of involvement. As said before, the base of the skull is an unusual site of involvement and most of the temporal bone $\mathrm{ABCs}$ occur on the skull vault; therefore, in many patients, local swelling with or without pain is the chief complaint. Involvement of adjacent bony and neural structures is seen received

January 7, 2016

accepted

January 14, 2016

published online

June 8, 2016
DOI http://dx.doi.org/

10.1055/s-0036-1581990. ISSN 2277-954X. (c) 2016 Neurological Surgeons' Society of India
License terms

(ब) (1) $\circledast$ 


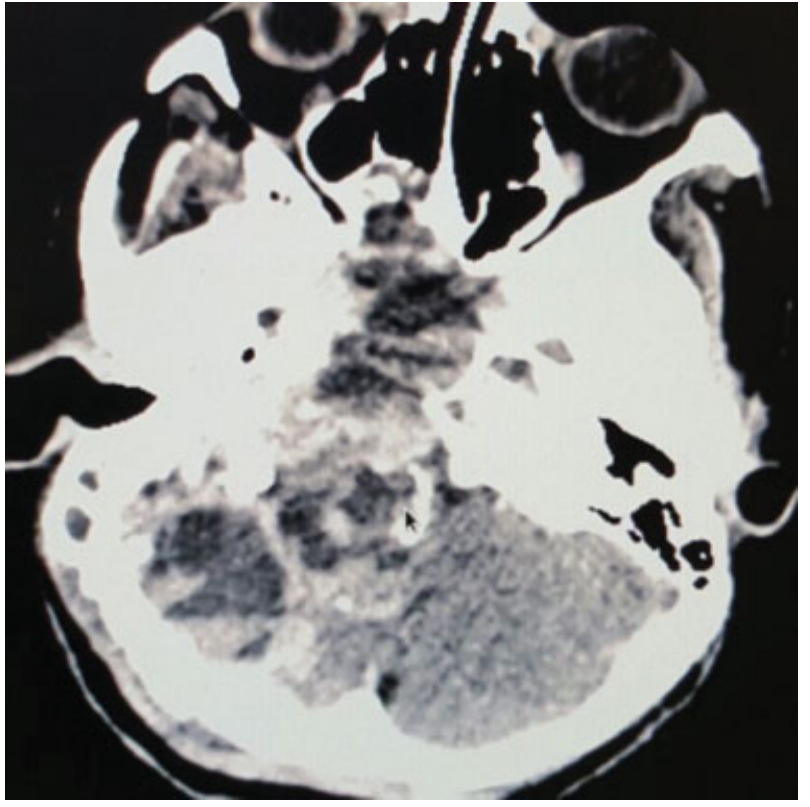

Fig. 1 Preoperative CT of the brain showing lesion extending from posterior fossa skull base up to clivus.

in some cases, but involvement of other cranial nerves is very rare. In our case, because of the special location, multiple cranial nerve involvement was present (7th-12th cranial nerve palsy) with signs of foramen jugular syndrome and hearing loss. A total of 30\% of ABCs are accompanied by other bony pathology and may be regarded as reactive phenomenon. The associated bony pathology included fibrous dysplasia, chondroblastoma, osteoblastoma, nonossifying fibroma, and giant cell tumors. ${ }^{2}$

It is postulated that the initiating process of the $A B C$ is the microcyst, which forms as a result of intercellular edema in a primary lesion with loose, unsupported stroma. Rupture of vessels into the microcysts introduces blood under hemodynamic pressure. With little resistance provided by the stroma, the blood spaces resorb the surrounding bone and

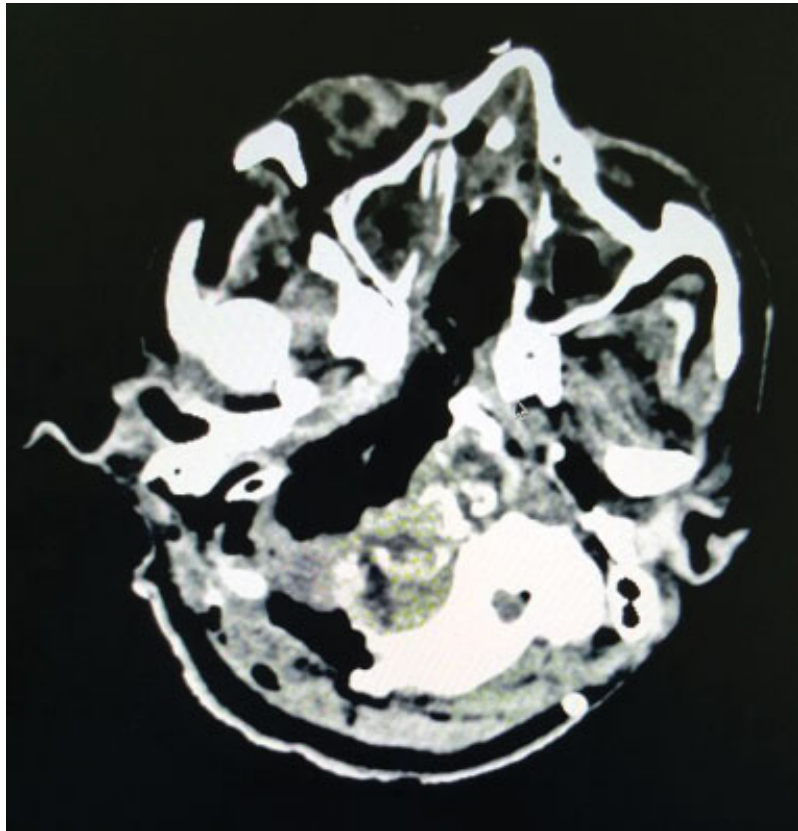

Fig. 3 Postoperative CT scan showing near-total excision with minimal residual lesion.

lift the periosteum, which produces a thin shell of new bone. Different theories about several vascular malformations exist; these include arteriovenous fistulas and venous blockage. The vascular lesions then cause increased pressure, expansion, erosion, and resorption of the surrounding bone. The malformation is also believed to cause local hemorrhage that initiates the formation of reactive osteolytic tissue. ${ }^{3}$

Most primary $A B C s$ demonstrate a $\mathrm{t}(16 ; 17)$ (q22; p13) fusion of the TRE17/CDH11-USP6 oncogene. This fusion leads to increased cellular cadherin- 11 activity that seems to arrest osteoblastic maturation in a more primitive state. This process may be the neoplastic driving force behind primary $\mathrm{ABCs}$ as opposed to secondary ABCs, which seem to occur reactively as a result of another underlying disease process. ${ }^{4}$
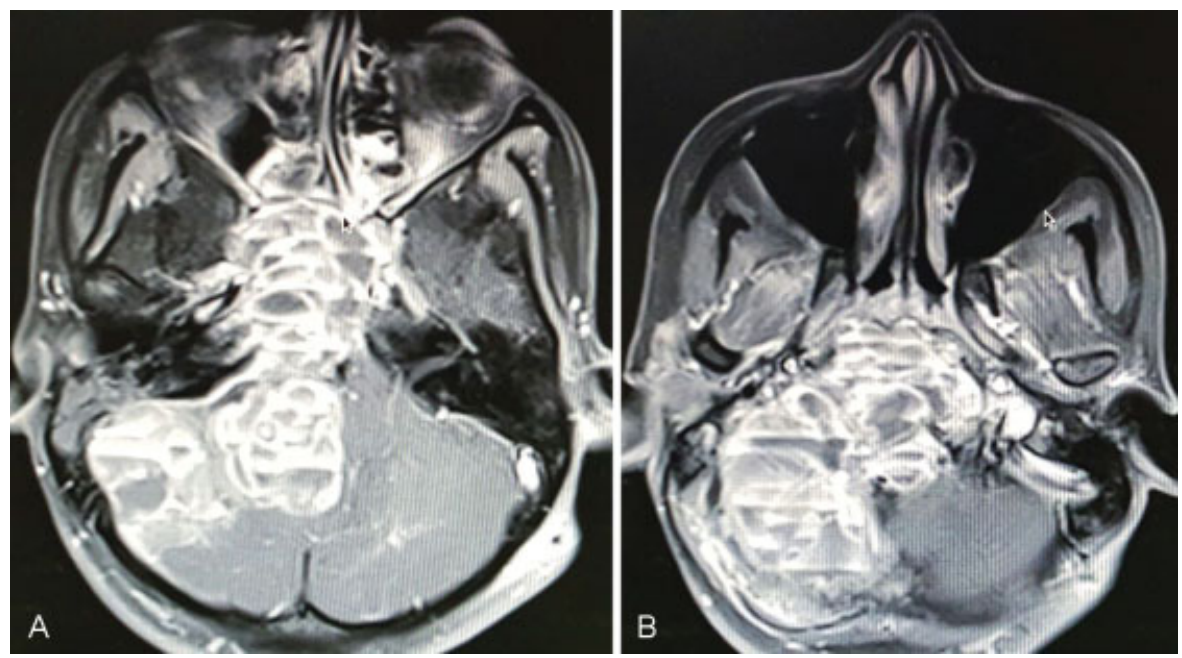

Fig. 2 (A, B) Preoperative MRI of the brain with contrast. 
On imaging, CT scan often exhibits soap bubble appearance for the multicystic mass and demarcates thinning of bony cortex with infrequent new bone formation around the lesions. On MRI, these lesions manifest with multiple fluid levels with peripheral rim enhancement. Other lesions such as telangiectatic osteosarcoma, giant cell granuloma, and benign fibro-osseous lesions also have been reported to have similar multiple fluid level appearance on the MRI images. ${ }^{1,2}$

Histologically, the lesion is characterized by the presence of multiple loculations, filled with venous blood and lined by spindle-shaped fibroblasts and with scattered multinucleated giant cells and stromal cells.

Treatment of the skull base ABC is by surgery, complete excision when possible, or partial resection when total removal cannot be accomplished. Total excision is the perfect treatment of $A B C$, which could be curative. However, in some patients treatment of skull base involvement with total excision is difficult and radiotherapy has been recommended. Preoperative embolization may reduce the tumor size and decrease intraoperative bleeding. Other modalities of treatment for $A B C s$ include sclerotherapy, embolization, radiotherapy, simple curettage, surgical excision, or some combination of these methods. Percutaneous sclerotherapy offers the least invasive initial therapeutic option when compared with surgery or endovascular strategies reserved for resistant lesions. When the surgical decompression is hazardous (possible damage to neural structures, massive hemorrhage) or in high-risk patients, sclerotherapy alone can be used at $A B C$ of cranium. Curettage is successfully used to treat ABCs. However, recurrence rates after curettage are between 21 and $50 \%$ in cases with cranial $A B C$ s treated. Surgical excision is the ideal treatment of choice for ABCs. Our patient underwent near-total excision followed by radiotherapy for residual lesion. $^{1,3}$

\section{Conclusion}

Although $A B C$ is a benign lesion, its unusual location and unusual presentation with multiple cranial nerves not reported in the literature make our case interesting.

\section{References}

1 Lee YC, Ho JT, Lin WC, Lui CC. Aneurysmal bone cyst of skull base: a case report and review of literature. Zhonghua Fang She Xue Za Zhi 2010;35:33-38

2 Aghaghazvini L, Sedighi N, Karami P, Yeganeh O. Skull base aneurysmal bone cyst presented with foramen jugular syndrome and multi-osseous involvement. Iran J Radiol 2012; 9(3):157-160

3 Purohit A, Chopra S, Sinha VD, Dharker SR. Aneurysmal bone cyst of the temporal bone: a case report. Neurol India 2002;50(4): 511-513

4 Oliveira AM, Perez-Atayde AR, Inwards CY, et al. USP6 and CDH11 oncogenes identify the neoplastic cell in primary aneurysmal bone cysts and are absent in so-called secondary aneurysmal bone cysts. Am J Pathol 2004;165(5):1773-1780 